\title{
A multicenter randomized controlled trial of surgery alone or surgery with atrial natriuretic peptide in lung cancer surgery: study protocol for a randomized controlled trial
}

\author{
Takashi Nojiri ${ }^{1,2,3^{*}}$ D, Haruko Yamamoto ${ }^{4}$, Toshimitsu Hamasaki ${ }^{5}$, Kaori Onda ${ }^{4}$, Kikuko Ohshima ${ }^{5}$, Yasushi Shintani ${ }^{2}$, \\ Meinoshin Okumura ${ }^{2}$, Kenji Kangawa ${ }^{1 *}$ on behalf of the Japan Human Atrial Natriuretic Peptide for Lung Cancer \\ Surgery (JANP Study) Group
}

\begin{abstract}
Background: Postoperative cancer recurrence is a major problem following curative surgery. In a previous retrospective study of lung cancer surgery, we reported that administration of atrial natriuretic peptide (ANP) during the perioperative period reduced postoperative recurrence. We demonstrated that ANP inhibited the adhesion of cancer cells to vascular endothelium as a vasoprotective action. The objective of this study is to evaluate the effects of ANP on the incidence of postoperative cancer recurrence in lung cancer surgery.

Methods/design: The present study is a multicenter, randomized trial with two parallel groups of patients with lung cancer comparing surgery alone and surgery with ANP administration for 3 days during the perioperative period. A total of 500 patients will be enrolled from 10 Japanese institutions. The primary endpoint is 2-year relapse-free survival (RFS). The secondary endpoints are 2-year cancer-specific RFS, 5-year RFS, overall survival, the incidence of postoperative complications, and the completion rate of ANP treatment.
\end{abstract}

Discussion: The principal question addressed in this trial is whether ANP with its vasoprotective action can reduce cancer recurrence following lung cancer surgery.

Trial registration: UMIN Clinical Trials Registry identifier: UMIN000018480. Registered on 31 July 2015.

Keywords: Lung cancer surgery, Atrial natriuretic peptide, Perioperative care, Cancer recurrence

\section{Background}

Cancer recurrence following curative surgery is a major problem after most cancer treatments. More than $50 \%$ of patients with resectable non-small cell lung cancer (NSCLC) will have recurrence after curative surgery [1]. Although complete surgical resection is currently considered the best option for cure in patients with most solid tumors, it is possible that surgical trauma itself influences the development of early recurrence [2-4]. First, handling

\footnotetext{
*Correspondence: nojiri@ri.ncvc.go.jp; kangawa@ri.ncvc.go.jp

${ }^{1}$ Department of Biochemistry, National Cerebral and Cardiovascular Center Research Institute, 5-7-1, Fujishirodai, Suita-city, Osaka 565-8565, Japan

Full list of author information is available at the end of the article
}

of the tumor during resection can provoke detachment of tumor cells, and the amount of circulating tumor cells is enhanced during resection of the primary tumor $[5,6]$. We previously reported that the presence of circulating tumor cells in the pulmonary vein during lung cancer surgery could be a prognostic indicator for early cancer recurrence [7]. Second, surgical trauma provokes a severe inflammatory reaction. There is emerging evidence to suggest that systemic inflammation can accelerate the adhesion of circulating tumor cells to the vascular endothelium of distant organs, which is a first step during extravasation in hematogenous metastasis $[8,9]$. Although most circulating tumor cells disappear rapidly [10], it is possible that residual 
cancer cells could attach more efficiently to the vascular endothelium by the release of inflammatory cytokines during the perioperative period $[8,9]$. Because no prophylactic strategy for cancer recurrence during the perioperative period has been established, the development of effective treatment is desirable.

We identified human atrial natriuretic peptide (ANP) as a diuretic/natriuretic and vasodilating hormone in 1984 [11]. ANP binds specifically to the guanylyl cyclase A receptor and has biological functions, including diuresis and inhibition of the renin-angiotensin-aldosterone system, as well as anti-inflammatory and antifibrotic actions $[12,13]$. In the clinical setting, ANP has been used to treat patients with acute heart failure in Japan since 1995. We have previously reported that the perioperative administration of ANP had a prophylactic effect on postoperative cardiopulmonary complications by attenuating the operationinduced inflammation in patients who underwent curative surgery for lung cancer [14-16]. Recently, we showed that cancer recurrence after curative surgery was significantly lower in ANP-treated patients than in control patients (surgery alone) [17]. We found that ANP inhibited the adhesion of cancer cells to vascular endothelium by suppressing E-selectin expression induced by inflammation [17]. On the basis of our previous findings, we planned a multicenter, phase II randomized controlled trial to confirm the effects of ANP on preventing postoperative recurrence following lung cancer surgery.

\section{Methods/design \\ Purpose}

The purpose of the present study is to evaluate the effects of ANP on reducing postoperative cancer recurrence in patients with lung cancer undergoing curative surgery.

\section{Study setting}

The study is an investigator-initiated, multi-institutional, two-arm, open-label, phase II randomized trial. The flowchart of the trial is shown in Fig. 1. The Standard Protocol Items: Recommendations for Interventional Trials (SPIRIT) 2013 checklist is given in Additional file 1.

\section{Endpoints}

The primary endpoint of this study is the 2-year relapsefree survival (RFS) rate after curative surgery for lung cancer. The secondary endpoints are 2-year cancerspecific RFS, 5-year RFS, 5-year overall survival, the rate of patients who complete ANP treatment, and the incidence of postoperative complications.

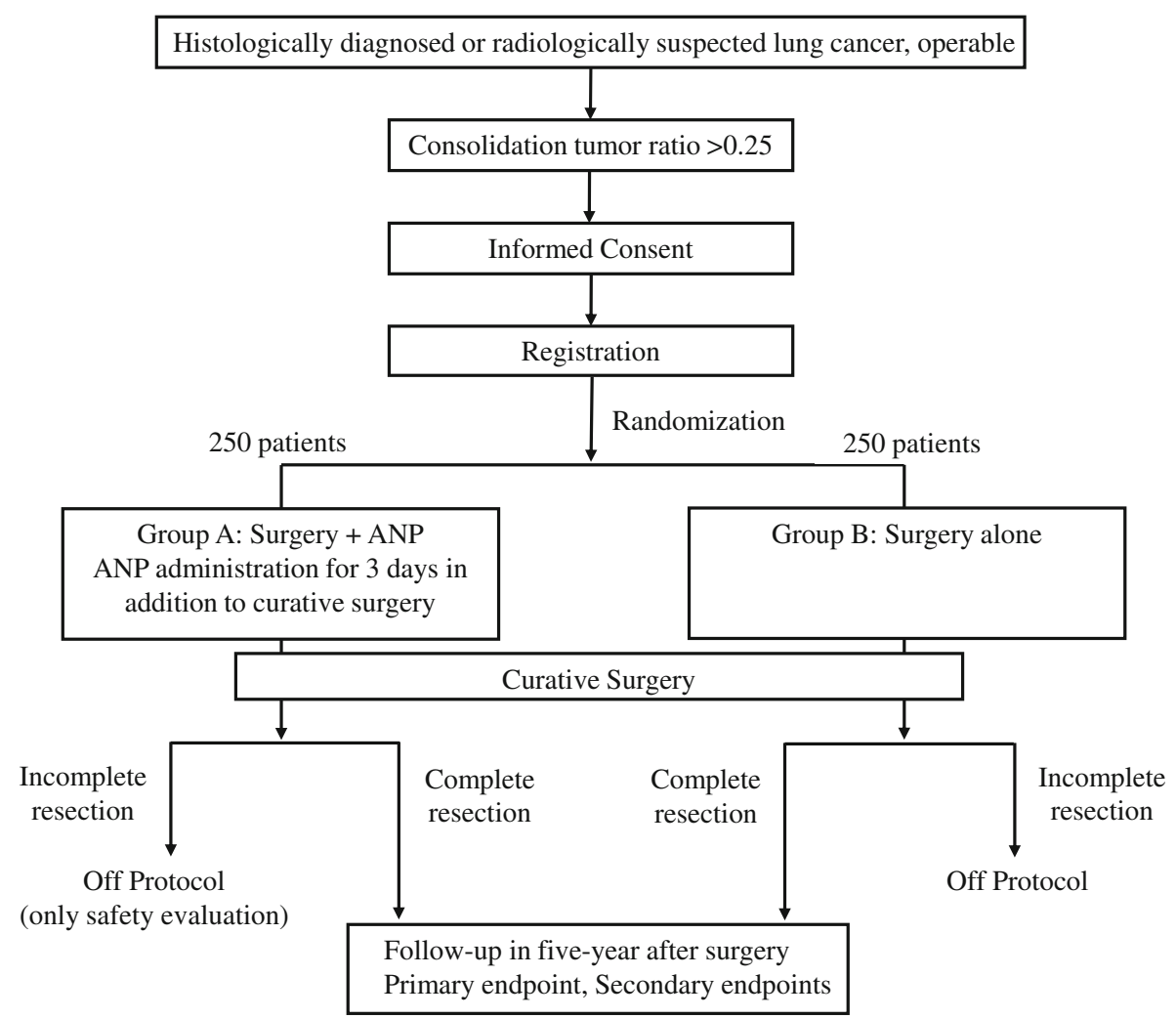

Fig. 1 Consolidated Standards of Reporting Trials (CONSORT) flowchart. ANP Atrial natriuretic peptide 


\section{Patient selection}

The tumors are staged according to the seventh edition of the Union for International Cancer Control TNM Classification of Malignant Tumours [18, 19]. Inclusion criteria are as follows:

1. NSCLC is suspected.

2. Radiologically diagnosed invasive lung tumor with a consolidation/tumor ratio $>0.25$ is visualized by thin-section computed tomography (CT).

3. Complete resection including mediastinal lymph node dissection is planned.

4. Patients must not have synchronous or metachronous (within 5 years) malignancies, except for carcinoma in situ or mucosal tumors curatively treated with local therapy.

5. Patients must be aged 20 years or older.

6. Eastern Cooperative Oncology Group performance status must be $0-2$.

7. Organ function must be sufficient (leukocyte count $\geq 1500 / \mathrm{ml}$, platelet count $\geq 1.0 \times 10^{5} / \mathrm{ml}$, hemoglobin $\geq 8.0 \mathrm{~g} / \mathrm{dl}$, total bilirubin $\leq 1.5 \mathrm{mg} / \mathrm{dl}$, aspartate aminotransferase $\leq 100 \mathrm{IU} / \mathrm{L}$, alanine aminotransferase $\leq 100 \mathrm{IU} / \mathrm{L}$, peripheral arterial oxygen saturation on room air $\geq 92 \%$ ).

8. Written informed consent is provided by the patient.

\section{Exclusion criteria}

The exclusion criteria are as follows:

1. Dominant pure ground-glass opacity (GGO) lesion (radiological noninvasive lung tumor with consolidation/tumor ratio $\leq 0.25$ visualized by thin-section CT)

2. Active concurrent malignant diseases

3. Pregnant, lactating, or potentially pregnant

4. Mental disorders that may affect the ability or willingness to provide informed consent or abide by the study protocol

5. Systemic steroids or immunosuppressive agent medication

6. Uncontrollable infectious disease, autoimmune disease, or other severe comorbidities

7. History of right ventricular infarction

8. Severe hypotension

9. Inappropriate for enrollment based on the judgment of the investigator

\section{Registration}

Eligible patients are registered and randomly assigned to either the surgery with ANP group or the surgeryalone group by the covariate-adaptive randomization method (Pocock-Simon procedure) including sex (male/ female), age ( $<70 / \geq 70$ years), clinical stage (IA/IB/ $\geq \mathrm{II})$, CT findings (including GGO/not including GGO), and institution as covariates and strata. The randomization ratio is 1:1. Both patients and investigators are open to treatment allocation. The Clinical Study Data Collecting System is used for patient registration and randomization, and the Research Electronic Data Capture $\left(\right.$ REDCap $\left.^{\mathrm{mm}}\right)$ system is used for data management. Enrollment was started in September 2015 and scheduled to continue for 2 years.

\section{Treatment methods}

Treatment flow is shown in Fig. 1. The patients enrolled in this study receive surgery alone or surgery with ANP (group A, curative surgery with ANP; group B, curative surgery alone).

In both groups, the surgical procedures undertaken include segmentectomy, lobectomy, or pneumonectomy with systematic node dissection in open thoracotomy or video-assisted thoracic surgery. Standard systematic node dissection (ND2) includes complete removal of the hilar and mediastinal nodes.

In group A, human ANP (Daiichi-Sankyo Pharmaceutical Inc., Tokyo, Japan) is continuously infused intravenously at $0.025 \mu \mathrm{g} \cdot \mathrm{kg}^{-1} \cdot$ minute $^{-1}$ for $72 \mathrm{~h}$ beginning more than $2 \mathrm{~h}$ before the start of surgery. In group B, there is no agent used in addition to curative surgery. The protocol treatment is to be stopped if curative surgery is not performed.

\section{Follow-up}

After curative resection, the information regarding postoperative complications within 30 days following surgery is recorded and sent to the data center. The incidence of postoperative complications is one of the secondary endpoints. All patients are followed with scheduled examinations, including a physical examination, serum biochemistry testing, chest $\mathrm{x}$-ray, contrast-enhanced chest and abdominal CT, contrast-enhanced brain magnetic resonance imaging (MRI), and bone scintigraphy to detect postoperative recurrence for 5 years. Fluorodeoxyglucosepositron emission tomography (FDG-PET) can replace bone scintigraphy. Chest and abdominal CT are performed every 6 months following surgery. Brain MRI and bone scintigraphy or FDG-PET are performed every year following surgery. The schedule of this trial is shown in Fig. 2.

\section{Radiological assessment}

Radiographic reviews for the development of postoperative recurrence are performed by the independent central image reading board, which consists of three certified radio-oncologists. All board members are blinded to treatment allocation. All films, including chest and abdominal CT, brain MRI, and bone scintigraphy or FDG-PET scans, are reviewed regularly by the board. 


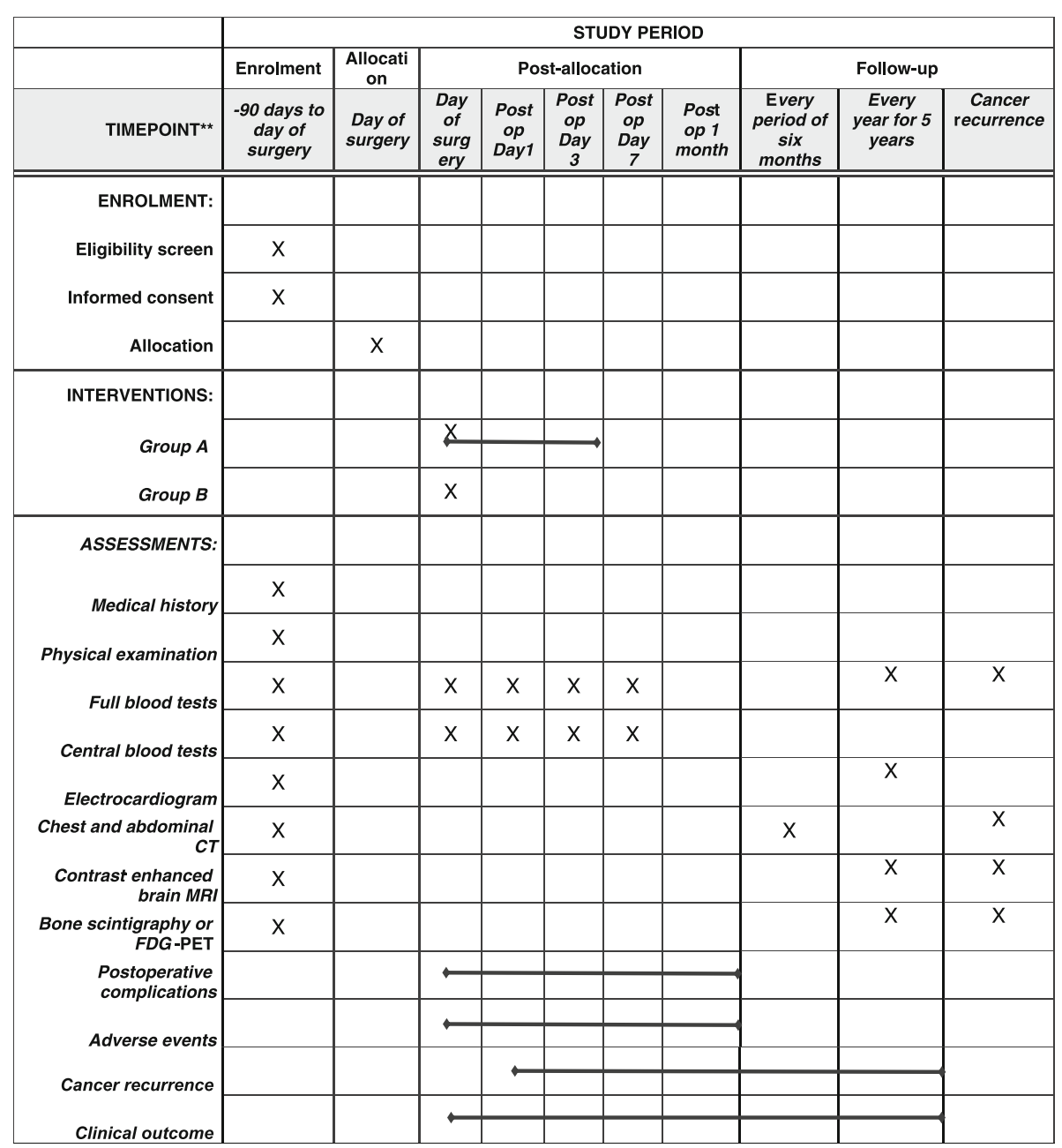

Fig. 2 Schedule of enrollment, interventions, and assessments for the Japan Human Atrial Natriuretic Peptide for Lung Cancer Surgery Study. CT Computed tomography, FDG-PET Fluorodeoxyglucose-positron emission tomography, MRI Magnetic resonance imaging

\section{Statistical methods}

The present study is a phase II randomized trial to evaluate the efficacy and safety of ANP administration during lung cancer surgery. This study is designed primarily to evaluate the 2-year RFS in both groups and to demonstrate whether ANP prevents postoperative recurrence within 2 years after curative surgery. In the previous study [17], the hazard ratio for the 2-year RFS was estimated to be 0.28 (95\% confidence interval $0.09-0.87$ ) in curative surgery with ANP compared with curative surgery alone. Because this previous observational study was retrospective, we conservatively assumed the hazard ratio to be 0.50 in the present study as the upper limit of the $70 \%$ confidence interval of the previous study. To evaluate the superiority of curative surgery with ANP (group A) over curative surgery alone (group B), the total sample size was calculated as 409 patients (95 events in total are expected), assuming $70 \%$ RFS in group B with $90 \%$ power at a onesided alevel of $2.5 \%$ using a log-rank test, assuming an expected accrual period of 2 years and a follow-up period of 2 years. A total of 500 patients will be recruited into the study, accounting for patients lost to follow-up, such as for benign tumors or incomplete resection.

Analyses will be done on the basis of the full analysis set (FAS). Based on the intention-to-treat (ITT) principle, the FAS includes all randomized subjects without major protocol violations. An ITT-based or per-protocol based analysis will be done to assess the robustness of the conclusions derived from the FAS-based analysis. Patient demographic data will be analyzed descriptively; categorical variables will be assessed with the chi-square test or Fisher's exact test, whereas continuous variables will be assessed with Student's $t$ test or the Wilcoxon rank-sum test, as appropriate. The survival curves for 2-year RFS (primary endpoint) and the 2-year cancer-specific RFS, 5year RFS, and overall survival (secondary endpoints) will be estimated using the Kaplan-Meier method and compared between the two groups by the log-rank test. The 
hazard ratio with its $95 \%$ confidence interval will be calculated using a proportional hazards model. The proportional hazards assumption will be investigated graphically, with a test based on Schoenfeld residuals. Safety data will be analyzed descriptively for the treated set, which consists of all randomized patients who receive at least one study treatment. All reported $P$ values will be two-sided. The statistical analysis plan, which includes more technical and detailed elaboration of the principal features stated in the protocol, will be prepared separately and finalized before database-locking. All statistical analyses will be conducted at the data center in the National Cerebral and Cardiovascular Research Center.

\section{Centralized monitoring and the Data and Safety Monitoring Committee}

Centralized monitoring is conducted for early identification and mitigation of data quality risk issues that may compromise the validity of the study results and for better assessment of patient safety. Also, the Data and Safety Monitoring Committee (DSMC) will independently review the report of trial monitoring regarding the efficacy and safety data derived from this study. On the basis of monitoring, the DSMC could consider early termination of a treatment regimen if a treatment-related death were to occur in group A during enrollment. The protocol compliance, safety, and on-schedule study progress are also monitored by the DSMC.

\section{Participating institutions}

The participating institutions in the study are Osaka University Hospital, The University of Tokyo Hospital, Hokkaido University Hospital, National Cancer Center Hospital East, Yamagata University Hospital, Kobe University Hospital, National Hospital Organization Toneyama Hospital, Osaka Medical Center for Cancer and Cardiovascular Diseases, Osaka Prefectural Medical Center for Respiratory and Allergic Diseases, and Yamagata Prefectural Central Hospital.

\section{Discussion}

To the best of our knowledge, this study is the first multicenter randomized clinical trial to examine the vasoprotective effects of ANP on cancer recurrence following curative surgery in patients with lung cancer. Surgical procedures may induce postoperative complications in the acute phase and cancer recurrence in the chronic phase after surgery by releasing inflammatory cytokines $[2,3]$. Vascular endothelial cells that become inflamed during the perioperative period can easily adhere to immune cells or circulating tumor cells, resulting in postoperative complications or cancer recurrence $[8,9]$. ANP has an anti-inflammatory action by reducing E-selectin levels in vascular endothelial cells [17]. In this study, we will be able to prospectively evaluate the effects of ANP on preventing both postoperative complications in the acute phase and cancer recurrence in the chronic phase after surgery. Surgical specimen and blood samples during the perioperative period will be collected to evaluate the mechanism of the effects of ANP, including serum soluble E-selectin levels.

Because the target of ANP is considered to be the vascular endothelium, this treatment can be used for all types of cancer surgery in principle. Most chemotherapy cannot be used during the perioperative period to reduce the incidence of cancer recurrence, owing to cytotoxicity. However, ANP has been proven not to cause severe adverse effects or hypotension on the basis of its use in patients with acute heart failure in Japan for over 20 years [20]. The results of this study will provide clinically valuable information for future cancer treatment.

\section{Trial status}

We are in the process of recruiting patients.

\section{Additional file}

Additional file 1: SPIRIT 2013 checklist: recommended items to address in a clinical trial protocol and related documents. (DOC $121 \mathrm{~kb}$ )

\section{Abbreviations \\ ANP: Atrial natriuretic peptide; CONSORT: Consolidated Standards of Reporting Trials; CT: Computed tomography; DSMC: Data and Safety Monitoring Committee; FAS: Full analysis set; FDG-PET: Fluorodeoxyglucose- positron emission tomography; GGO: Ground-glass opacity; ITT: Intention to treat; JANP: Japan Human Atrial Natriuretic Peptide for Lung Cancer Surgery Study; MRI: Magnetic resonance imaging; ND2: Standard systematic node dissection; NSCLC: Non-small cell lung cancer; REDCap: Research Electronic Data Capture; RFS: Relapse-free survival; SPIRIT: Standard Protocol Items: Recommendations for Interventional Trials}

\section{Acknowledgements \\ JANP Study Group members and participating centers:}

1. Dr. Meinoshin Okumura, Osaka University Hospital, Osaka, Japan; meinosin@thoracic.med.osaka-u.ac.jp

2. Dr. Jun Nakajima, The University of Tokyo Hospital, Tokyo, Japan; nakajimatho@h.u-tokyo.ac.jp

3. Dr. Masahiro Tsuboi, National Cancer Center Hospital East, Chiba, Japan; mtsuboi@za2.so-net.ne.jp

4. Dr. Yasuhiro Hida, Hokkaido University Hospital, Hokkaido, Japan;

yhida@med.hokudai.ac.jp

5. Dr. Yoshimasa Maniwa, Kobe University Hospital, Hyogo, Japan; maniwa@med.kobe-u.ac.jp

6. Dr. Hiroyuki Oizumi, Yamagata University Hospital, Yamagata, Japan; hohizumi@med.id.yamagata-u.ac.jp

7. Dr. Masahiko Higashiyama, Osaka Medical Center for Cancer and Cardiovascular Diseases, Osaka, Japan; higasiyama-ma@mc.pref.osaka.jp 8. Dr. Yukiyasu Takeuchi, National Hospital Organization Toneyama Hospital, Osaka, Japan; ytakeuch@toneyama.go.jp

9. Dr. Yoshihisa Kadota, Osaka Prefectural Medical Center for Respiratory and Allergic Diseases, Osaka, Japan; kadotay@ra.opho.jp

10. Dr. Satoshi Shiono, Yamagata Prefectural Central Hospital, Yamagata, Japan; sshiono@ypch.gr.jp.

\section{Funding}

This study is supported by the Japan Agency for Medical Research and Development (K067) and Shionogi \& Co., Ltd. (C283). The funding bodies 
had no role in the study design and implementation, the writing of the report, or the decision to submit the manuscript for publication.

\section{Availability of data and materials}

To avoid bias of the analysis, the dataset supporting the conclusions of this article will not be available until the final report of this trial is published.

\section{Authors' contributions}

TN conceived of the study and wrote the manuscript. HY and KOn conceived of and coordinated the study. TH and KOh performed statistical analysis and provided the biostatistical study design. YS and MO conceived of and supervised the study. KK conceived of and supervised the study and is the grant holder. All authors read and approved the final manuscript.

\section{Competing interests}

$\mathrm{TN}, \mathrm{MO}$, and $\mathrm{KK}$ have the filed patent related to ANP for the treatment of cancer metastasis with Shionogi \& Co., Ltd., which has part of the right to apply for the patent transferred from one of the original applicants, Daiichi-Sankyo Pharmaceutical Inc. (PCT/JP2012/054841). Shionogi \& Co., Ltd., was not involved in the study design and was not involved in the collection, analysis, or interpretation of data; in the writing of the manuscript; or in the decision to submit the manuscript for publication. The other authors declare that they have no competing interests.

\section{Consent for publication}

Not applicable.

\section{Ethics approval and consent to participate}

The final protocol was approved by the Japanese Advanced Medical Care B program in June 2015 (Japanese Advanced Medical Care B program number 60), and patient enrollment began in September 2015. Before enrollment of the patients, the study protocol was approved by each institutional review board of all participating centers. This study complies with the Declaration of Helsinki. Written informed consent will be obtained from all patients before they are recruited. Personal information about potential and enrolled participants will remain confidential, and data will be de-identified using participant numbers. This trial is registered in the UMIN Clinical Trials Registry with the identifier UMIN000018480 (http://www.umin.ac.jp/ctr/index-j.htm). The institutional review board of National Cerebral and Cardiovascular Center approved the protocol (M26-095). Before enrollment of the patients, this study was approved by the institutional review boards of Osaka University Hospital (14236-2), The University of Tokyo Hospital (P2015015-11X), Hokkaido University Hospital (015-0217), National Cancer Center Hospital East (2015-198), Yamagata University Hospital (101), Kobe University Hospital (270021), National Hospital Organization Toneyama Hospital (1523), Osaka Medical Center for Cancer and Cardiovascular Diseases (1509245094), Osaka Prefectural Medical Center for Respiratory and Allergic Diseases (732), and Yamagata Prefectural Central Hospital (24).

\section{Publisher's Note}

Springer Nature remains neutral with regard to jurisdictional claims in published maps and institutional affiliations.

\section{Author details}

${ }^{1}$ Department of Biochemistry, National Cerebral and Cardiovascular Center Research Institute, 5-7-1, Fujishirodai, Suita-city, Osaka 565-8565, Japan. ${ }^{2}$ Department of General Thoracic Surgery, Osaka University Graduate School of Medicine, 2-2 (L5) Yamadaoka, Suita-City, Osaka 565-0871, Japan. ${ }^{3}$ Department of General Thoracic Surgery, National Hospital Organization Toneyama Hospital, 5-1-1 Toneyama, Toyonaka-City, Osaka 560-8552, Japan. ${ }^{4}$ Center for Advancing Clinical and Translational Sciences, National Cerebral and Cardiovascular Center, 5-7-1, Fujishirodai, Suita-city, Osaka 565-8565, Japan. ${ }^{5}$ Department of Data Science, National Cerebral and Cardiovascular Center, 5-7-1, Fujishirodai, Suita-city, Osaka 565-8565, Japan.

Received: 11 January 2017 Accepted: 4 April 2017

Published online: 20 April 2017

\section{References}

1. Mountain CF. Revisions in the International System for Staging Lung Cancer. Chest. 1997;111(6):1710-7.
2. Ferri LE, Law S, Wong KH, Kwok KF, Wong J. The influence of technical complications on postoperative outcome and survival after esophagectomy. Ann Surg Oncol. 2006;13(4):557-64.

3. ten Kate M, Hofland LJ, van Grevenstein WM, van Koetsveld PV, Jeekel J, van Eijck CH. Influence of proinflammatory cytokines on the adhesion of human colon carcinoma cells to lung microvascular endothelium. Int J Cancer. 2004;112(6):943-50.

4. Andalib A, Ramana-Kumar AV, Bartlett G, Franco EL, Ferri LE. Influence of postoperative infectious complications on long-term survival of lung cancer patients: a population-based cohort study. J Thorac Oncol. 2013;8(5):554-61.

5. van den Tol PM, van Rossen $\mathrm{EE}$, van Eijck $\mathrm{CH}$, Bonthuis $\mathrm{F}$, Marquet $\mathrm{RL}$, Jeeke $H$. Reduction of peritoneal trauma by using nonsurgical gauze leads to less implantation metastasis of spilled tumor cells. Ann Surg. 1998;227(2):242-8.

6. Yamaguchi K, Takagi Y, Aoki S, Futamura M, Saji S. Significant detection of circulating cancer cells in the blood by reverse transcriptase-polymerase chain reaction during colorectal cancer resection. Ann Surg. 2000;232(1):58-65.

7. Funaki S, Sawabata N, Nakagiri T, Shintani Y, Inoue M, Kadota Y, et al. Novel approach for detection of isolated tumor cells in pulmonary vein using negative selection method: morphological classification and clinical implications. Eur J Cardiothorac Surg. 2011;40(2):322-7.

8. Giavazzi R, Foppolo M, Dossi R, Remuzzi A. Rolling and adhesion of human tumor cells on vascular endothelium under physiological flow conditions. J Clin Invest. 1993;92(6):3038-44.

9. McDonald B, Spicer J, Giannais B, Fallavollita L, Brodt P, Ferri LE. Systemic inflammation increases cancer cell adhesion to hepatic sinusoids by neutrophil mediated mechanisms. Int J Cancer. 2009:125(6):1298-305.

10. Stott SL, Lee RJ, Nagrath S, Yu M, Miyamoto DT, Ulkus L, et al. Isolation and characterization of circulating tumor cells from patients with localized and metastatic prostate cancer. Sci Transl Med. 2010;2(25):25ra23.

11. Kangawa K, Matsuo H. Purification and complete amino acid sequence of a-human atrial natriuretic polypeptide (a-hANP). Biochem Biophys Res Commun. 1984;118(1):131-9.

12. Kishimoto I, Tokudome T, Horio T, Garbers DL, Nakao K, Kangawa K Natriuretic peptide signaling via guanylyl cyclase (GC)-A: an endogenous protective mechanism of the heart. Curr Cardiol Rev. 2009:5(1):45-51.

13. Kasama S, Furuya M, Toyama T, Ichikawa S, Kurabayashi M. Effect of atrial natriuretic peptide on left ventricular remodelling in patients with acute myocardial infarction. Eur Heart J. 2008;29(12):1485-94.

14. Nojiri T, Yamamoto $K$, Maeda $H$, Takeuchi $Y$, Funakoshi $Y$, Inoue $M$, et al. Effect of low-dose human atrial natriuretic peptide on postoperative atrial fibrillation in patients undergoing pulmonary resection for lung cancer: a double-blind, placebo-controlled study. J Thorac Cardiovasc Surg. 2012; 143(2):488-94

15. Nojiri $T$, Inoue $M$, Yamamoto $K$, Maeda $H$, Takeuchi $Y$, Funakoshi $Y$, et al. Effects of low-dose human atrial natriuretic peptide for preventing post-operative cardiopulmonary complications in elderly patients undergoing pulmonary resection for lung cancer. Eur J Cardiothorac Surg. 2012;41(6):1330-4.

16. Nojiri $T$, Inoue M, Maeda H, Takeuchi Y, Sawabata N, Shintani $Y$, et al. Low-dose human atrial natriuretic peptide for the prevention of postoperative cardiopulmonary complications in chronic obstructive pulmonary disease patients undergoing lung cancer surgery. Eur J Cardiothorac Surg. 2013;44(1):98-103.

17. Nojiri T, Hosoda H, Tokudome T, Miura K, Ishikane S, Otani K, et al. Atrial natriuretic peptide prevents cancer metastasis through vascular endothelial cells. Proc Natl Acad Sci U S A. 2015;112(13):4086-91.

18. Goldstraw P, Crowley J, Chansky K, Giroux DJ, Groome PA, Rami-Porta R, et al. The IASLC Lung Cancer Staging Project: proposals for the revision of the TNM stage groupings in the forthcoming (seventh) edition of the TNM Classification of Malignant Tumours. J Thorac Oncol. 2007;2(8):706-14.

19. Sobin LH, Gospodarowicz MK, Wittekind C, editors. TNM classification of malignant tumours. 7th ed. Hoboken: John Wiley \& Sons; 2010.

20. Nomura F, Kurobe N, Mori Y, Hikita A, Kawai M, Suwa M, et al. Multicenter prospective investigation on efficacy and safety of carperitide as a first-line drug for acute heart failure syndrome with preserved blood pressure: COMPASS: Carperitide Effects Observed through Monitoring Dyspnea in Acute Decompensated Heart Failure Study. Circ J. 2008;72(11):1777-86. 